\title{
PERFIL DAS CONDIÇÕES DE SEGUIMENTO TERAPÊUTICO EM PORTADORES DE HIPERTENSÃO ARTERIAL ${ }^{a}$
}

\author{
Survey of the follow-therapeutic patients with hypertension \\ Perfil de las condiciones del seguimiento terapéutico en pacientes con hipertensión
}

\author{
Allyne Fortes Vitor ${ }^{1}$ \\ João Dennys Pinheiro Vasconcelos ${ }^{4}$
}

\author{
Flávia Paula Magalhães Monteiro² \\ Marcos Venícios de Oliveira Lopes ${ }^{5}$
}

\author{
Huana Carolina Cândido Morais ${ }^{3}$ \\ Thelma Leite de Araujo ${ }^{6}$
}

\section{RESUMO}

A proposta foi investigar os aspectos comportamentais referentes ao seguimento da terapêutica farmacológica e não farmacológica e o grau de adesão ao tratamento anti-hipertensivo de um grupo específico. Estudo observacional descritivo com análise quantitativa, realizado no Centro de Referência da Assistência Social em Fortaleza-CE, de agosto/2008 a maio/2009. Dados obtidos de 49 indivíduos por entrevista e exame físico. Mais de 50\% dos participantes seguiam as terapêuticas não farmacológicas. Prevaleceram a terapia combinada ( $53 \%$ ) e as classes medicamentosas de diuréticos (72\%) e inibidores da enzima conversora de angiotensina (55\%). Muitos participantes (49\%) referiram reações adversas. Destas, as mais citadas foram poliúria e tontura (29\%). Quanto ao grau de adesão, a média correspondeu ao conceito não adesão leve. A avaliação dos comportamentos de seguimento terapêutico e a caracterização clínico-epidemiológica são necessárias para o enfermeiro planejar estratégias educativas. Ambas possibilitam ajustes no planejamento das intervenções, contribuindo para a melhor adesão terapêutica dos indivíduos.

Palavras-chave: Enfermagem. Hipertensão. Cooperação do Paciente.

\begin{abstract}
The purpose was to investigate the behavioral aspects on the follow-up of pharmacologic therapy and no-pharmacologic and the patient's compliance degree to treatment of Hypertension in a specific group. Observational descriptive study by quantitative analysis accomplished on the Reference Center of Social Assistant, Ceará, from August 2008 to May 2009. Data collected by the interview and physical examination, on 49 individuals. More than $50 \%$ of participants followed the non-pharmacological treatment, except the physical activity. It was prevailed the combination therapy (53\%), the use of diuretics (72\%) and angiotensin converting enzyme inhibitors (55\%). Almost half (49\%) reported adverse reactions, predominantly polyuria and dizziness (29\%). The mean of patient's compliance degree was corresponding to light noncompliance. The evaluation of the behavior about therapeutic follow-up and clinical-epidemiological characteristics are necessary to validate educational actions developed by nurses, allowing adjustments to plan of interventions, contributing to a better compliance to the treatment.
\end{abstract}

Keywords: Nursing. Hypertension. Patient Compliance.

\section{Resumen}

El propósito ha sido investigar los aspectos de comportamiento en el seguimiento de la terapia farmacológica y no-farmacológica y el grado de adhesión del paciente al tratamiento anti-hipertensión en un grupo específico. Estudio observacional descriptivo, mediante análisis cuantitativo, desarrollado en el Centro de Referencia de Asistencia Social, en Fortaleza, Ceará, de agosto/2008 hasta mayo/2009. Los datos han sido recogidos por entrevista y examen físico en 49 individuos. Más de $50 \%$ de los participantes siguió el tratamiento no-farmacológico. La terapia de combinación prevaleció (53\%) y las clases más expresivas de los medicamentos han sido los diuréticos (72\%) y los inhibidores de la enzima de conversión de angiotensina (55\%). Casi la mitad (49\%) reportaron reacciones adversas, sobretodo poliuria y mareos (29\%). El promedio del grado de adhesión al tratamiento correspondió a bajo. La evaluación de las características clínico-epidemiológica y la conducta sobre el seguimiento de la terapia son necesarias para validar las acciones educativas desarrolladas por enfermeras, lo que permite ajustes en la planificación de intervenciones y contribuye para una mejor adhesión al tratamiento.

Palabras-clave: Enfermería. Hipertensión. Cooperación del Paciente

\footnotetext{
'Enfermeira. Aluna do curso de Doutorado do Programa de Pós-graduação em Enfermagem da Universidade Federal do Ceará/UFC. Bolsista do CNPq vinculada ao Projeto Cuidado em Saúde Cardiovascular/CNPq. Fortaleza-CE. Brasil. E-mail: allynefortes@yahoo.com.br, ${ }^{2}$ Enfermeira. Aluna do curso de Doutorado do Programa de Pós-graduação em Enfermagem da Universidade Federal do Ceará/UFC. Vinculada ao Projeto Cuidado em Saúde Cardiovascular/ CNPq. Fortaleza-CE. Brasil. E-mail: flaviapmm@yahoo.com.br, ${ }^{3}$ Acadêmica de Enfermagem do $9^{\circ}$ semestre do Curso de Graduação em Enfermagem da Universidade Federal do Ceará. Bolsista do CNPq vinculado ao Projeto Cuidado em Saúde Cardiovascular/CNPq. Fortaleza-CE. Brasil. E-mail: huanacarolina@yahoo.com.br, ${ }^{4}$ Acadêmico de Enfermagem do $9^{\circ}$ semestre do Curso de Graduação em Enfermagem da Universidade Federal do Ceará. Bolsista do CNPq vinculado ao Projeto Cuidado em Saúde Cardiovascular/CNPq. Fortaleza-CE. Brasil. E-mail:dennys_vasconcelos@hotmail.com, ${ }^{5}$ Doutor em Enfermagem. Professor Adjunto do Departamento de Enfermagem da Universidade Federal do Ceará (UFC). Fortaleza-CE. Brasil. E-mail: marcos@ufc.br, ${ }^{6}$ Doutora em Enfermagem. Professora Associada do Departamento de Enfermagem da Universidade Federal do Ceará (UFC). Fortaleza-CE. Brasil. E-mail: thelmaaraujo2003@yahoo.com.br
} 


\section{INTRODUÇÃO}

A hipertensão arterial (HA) encontra-se entre as doenças crônicas não transmissíveis mais prevalentes entre as pessoas, como uma entidade clínica multifatorial, de caráter multígeno e que, na maioria dos casos, é assintomática. ${ }^{1}$ Por ser uma doença crônica, pode ser controlada, mas não curada. Portanto, requer tratamento por toda a vida. Seu controle, quando diminuem as complicações, está diretamente relacionado ao grau de adesão do paciente à terapêutica que Ihe é indicada. ${ }^{2}$

Compreende-se adesão ao regime terapêutico como 0 comportamento do paciente coincidente com as orientações prescritas pelos profissionais de saúde. Isso significa uma relação colaborativa entre as duas partes envolvidas no processo. $^{3}$

Adaptar-se ao tratamento constitui a tarefa mais difícil, e é responsável pela enorme resistência encontrada pelos profissionais para a adesão do paciente ao regime terapêutico. A não adesão é um grande obstáculo no controle da doença.

Por este motivo, tornam-se necessários investimentos em diferentes fatores, especificamente nos aspectos comportamentais, os quais poderão aumentar a adesão do paciente ao seguimento terapêutico. No entanto, estudos reportam que ainda existem obstáculos que impedem esta iniciativa, pois o grande desafio se encontra na dificuldade de envolver familiares/cuidadores e doentes como participantes deste processo de adesão à terapia direcionada aos pacientes hipertensos. ${ }^{4}$

Existem vários fatores a influenciar no grau de adesão do paciente ao seu tratamento anti-hipertensivo. Entre eles: 0 sexo, a idade, o grau de instrução, a condição socioeconômica, o estado civil, o conhecimento e as crenças sobre as doenças, o seu estilo de vida, a presença do paciente na unidade de saúde e o apoio familiar. Além disso, somam-se fatores relacionados ao tratamento farmacológico e não farmacológico, como quantidade, dosagem, horários, efeitos colaterais e custo dos medicamentos, mudança de hábitos seguidos de restrições alimentares, de lazer e de trabalho. Finalmente, as políticas de saúde, a disponibilidade de medicamentos no serviço de saúde, a facilidade de marcar consultas e a presença de uma equipe de saúde multidisciplinar são fatores decisivos na adesão ao tratamento. ${ }^{2}$

Como integrante fundamental da equipe de saúde, 0 enfermeiro assume a responsabilidade das ações do cuidado para a promoção da saúde e prevenção desta doença. Por meio do conhecimento científico e do seu papel de educador, ele tem a possibilidade de instrumentalizar o portador da doença para o tratamento, melhorando sua qualidade de vida. ${ }^{5}$

A partir desses argumentos, se discutirão as características sociodemográficas e clínico-epidemiológicas da clientela em tratamento anti-hipertensivo. Outros temas abordados referem-se ao tratamento farmacológico utilizado para o controle da doença, a presença de reações adversas destes medicamentos e o grau de adesão dos indivíduos às terapêuticas mediante a aplicação da escala proposta por Moreira (2003). ${ }^{6}$

Este estudo poderá representar contribuição para o conhecimento geral dos profissionais e para a construção de estratégias de atendimento capazes de aumentar a adesão ao tratamento. Para tal, identificará as medidas terapêuticas que representam maiores dificuldades no seguimento, e assim possibilitará amelhoria na qualidade de vida dos portadores dehipertensão arterial.

\section{METODOLOGIA}

Trata-se de estudo de natureza observacional descritiva, com análise quantitativa, realizado no Centro de Referência da Assistência Social (CRAS), na cidade de Fortaleza-CE no período de agosto de 2008 a maio de 2009. Mencionado centro é um espaço destinado ao atendimento socioassistencial da população, articulando os serviços disponíveis em cada localidade, bem como potencializando a rede de proteção social básica. Os participantes do estudo foram recrutados por período de tempo (nove meses) mediante a técnica de amostragem não probabilística por conveniência.

Dessa forma, foram incluídos os indivíduos que frequentavam o CRAS, de ambos os sexos, formado prioritariamente, mas não exclusivamente, por idosos; com diagnóstico médico de hipertensão arterial há mais de um ano e em tratamento farmacológico há pelo menos seis meses. Foram excluídos os indivíduos incapazes de responder ao questionário proposto. Utilizaram-se dois instrumentos para a coleta de dados. 0 primeiro foi destinado à investigação das condições sociodemográficas (procedência, sexo, idade, cor, estado civil, grau de escolaridade, atuação profissional, renda familiar mensal e constituição familiar), das condições clínico-epidemiológicas (estatura, peso, índice de massa corporal, circunferências abdominal e do quadril e pressão arterial). Incluíram-se também as informações relativas às medicações usadas no tratamento farmacológico para hipertensão arterial quanto à existência de efeitos adversos das drogas e dos comportamentos adotados em relação ao tratamento da hipertensão arterial (dieta, consumo de frutas everduras, hábitos de tabagismo e etilismo, controle do estresse, prática de atividade física, tipo, frequência e intensidade). Os dados referentes a este instrumento foram coletados com a utilização de entrevista e exame físico. 0 outro instrumento, aplicado no segundo encontro, foi elaborado e validado por Moreira (2003). ${ }^{6}$ Constitui-se de uma escala, a qual foi aplicada para avaliar a adesão ao tratamento da hipertensão arterial por meio de investigação de dez variáveis que compõem o tratamento anti-hipertensivo medicamentoso e de mudança do estilo de vida. A pontuação da escala de Moreira $(2003)^{6}$ possui valor mínimo de zero e valor máximo de dez, referentes às seguintes variáveis: consumo adequado de sal; consumo adequado de gordura; índice de massa corporal (IMC); abstinência do fumo; ausência de ingesta alcoólica; prática regular de exercícios físicos; 
enfrentamento eficaz do estresse; uso adequado do medicamento; comparecimento às consultas/período em que são marcadas na UBSF; controle da pressão arterial (PA). Esta escala permite classificar assim a adesão terapêutica: paciente ideal $(X=10)$, adesão terapêutica $(10>X \geq 9)$, não adesão leve $(9>X \geq 7)$, não adesão moderada $(7>X \geq 5)$, não adesão grave $(5>X \geq 3)$ e não adesão gravíssima $(3>X \geq 0)$.

Os valores de pressão arterial utilizados para avaliar a adesão foram as médias obtidas em três encontros determinados em diferentes momentos, seguindo-se as recomendações para a medida correta. ${ }^{1}$

Os dados foram compilados e organizados em planilhas do Excel. Em seguida, foram submetidos a testes estatísticos com auxílio do Software Statistical Package for Social Sciences (SPSS) versão 15.0. Para os testes utilizados, o nível de significância adotado foi de $5 \%(p<0,05)$. A análise foi realizada mediante uma abordagem estatística descritiva, para a qual foi feita a distribuição das frequências absolutas e relativas para as variáveis categóricas e das médias, desvio-padrão e percentis, para variáveis contínuas. Para a análise estatística foi realizado o teste de Kolmogorov-Smirnov, com vistas à verificação da normalidade dos dados numéricos. Para verificação da correlação entre variáveis numéricas foi calculado o coeficiente de correlação de Pearson.
Como exigido, o estudo atendeu às recomendações da Resolução 196/96 do Conselho Nacional de Saúde, referentes às pesquisas envolvendo seres humanos ${ }^{7}$, sendo avaliado e aprovado pelo Comitê de Ética em Pesquisa (CEPE) da Universidade Federal do Ceará, mediante protocolo n ${ }^{\circ} 89 / 08$. Ainda como recomendado, os dados somente foram coletados após assinatura do Termo de Consentimento Livre e Esclarecido pelo participante.

\section{RESULTADOS}

Participaram do estudo 49 portadores de hipertensão arterial, sendo a maioria, 38 (77,5\%), do sexo feminino, pertencentes à faixa etária de 50 a 85 anos, com média de 68,6 anos de idade. No inerente à composição familiar, 18 (36\%) deles residiam com companheiro, enquanto 31 (64\%) não residiam com companheiro, estando nesta categoria os solteiros, viúvos ou divorciados; dos clientes, $7(14,28 \%)$ moravam sozinhos. A média de pessoas por domicilio foi igual a 3,28. Quanto à escolaridade, 40 (82\%) eram analfabetos ou somente sabiam ler ou escrever, e apenas 5 (10\%) concluíram o ensino médio. Amédia foi de 4,1 anos de estudo, caracterizando o baixo grau de escolaridade. Como ocupação mais referida foi mencionada a de aposentado por 30 (61\%) pessoas, seguida pelade dona-do-lar, $10(21 \%)$. Arendafamiliar média foi de $R \$ 779,63$. Sobrea cor, a maioria dos indivíduos, $33(67 \%)$, considerou-se parda, enquanto 2 (4\%) afirmaram-senegros (Tabela 1).

Tabela 1: Distribuição dos participantes segundo as variáveis socioeconômicas. Centro de Referência em Assistência Social de Fortaleza-CE, 2009.

\begin{tabular}{|c|c|c|}
\hline Variáveis & $\mathrm{N}$ & $\%$ \\
\hline \multicolumn{3}{|l|}{ Sexo } \\
\hline Masculino & 11 & $22,5 \%$ \\
\hline Feminino & 38 & $77,5 \%$ \\
\hline Total & 49 & $100 \%$ \\
\hline \multicolumn{3}{|l|}{ Idade } \\
\hline 45 a 50 anos & 4 & $8 \%$ \\
\hline 56 a 60 anos & 3 & $6 \%$ \\
\hline 61 a 65 anos & 10 & $21 \%$ \\
\hline 66 a 70 anos & 12 & $25 \%$ \\
\hline 71 a 75 anos & 8 & $16 \%$ \\
\hline 76 a 80 anos & 10 & $20 \%$ \\
\hline 81 a 85 anos & 2 & $4 \%$ \\
\hline Total & 49 & $100 \%$ \\
\hline \multicolumn{3}{|l|}{ Cor Referida } \\
\hline Branca & 14 & $29 \%$ \\
\hline Parda & 33 & $67 \%$ \\
\hline Negra & 2 & $4 \%$ \\
\hline Total & 49 & $100 \%$ \\
\hline \multicolumn{3}{|l|}{ Estado Marital } \\
\hline Com companheiro & 18 & $36 \%$ \\
\hline Sem companheiro & 31 & $64 \%$ \\
\hline Total & 49 & $100 \%$ \\
\hline \multicolumn{3}{|l|}{ Grau de Escolaridade } \\
\hline Analfabeto & 10 & $20 \%$ \\
\hline Lê ou assina & 30 & $62 \%$ \\
\hline Fundamental Completo & 4 & $8 \%$ \\
\hline Médio Completo & 5 & $10 \%$ \\
\hline Total & 49 & $100 \%$ \\
\hline \multicolumn{3}{|l|}{ Ocupação } \\
\hline Aposentado & 30 & $61 \%$ \\
\hline Dona do Lar & 10 & $21 \%$ \\
\hline Outros & 9 & $18 \%$ \\
\hline Total & 49 & $100 \%$ \\
\hline \multicolumn{3}{|c|}{ Renda Familiar (em salários mínimos) } \\
\hline Até 1 & 24 & $49 \%$ \\
\hline De 1 a 2 & 11 & $22 \%$ \\
\hline De 2 a 3 & 6 & $13 \%$ \\
\hline Mais de 3 & 8 & $16 \%$ \\
\hline Total & 49 & $100 \%$ \\
\hline
\end{tabular}


No concernente aos dados antropométricos, observaram-se as seguintes médias: $28 \mathrm{~kg} / \mathrm{m}^{2}( \pm 3,9 \mathrm{~kg} /$ $\left.\mathrm{m}^{2}\right)$ para o índice de massa corporal; 0,9 $( \pm 0,1)$ referente ao RCQ (relação cintura-quadril); $98 \mathrm{~cm}( \pm 10 \mathrm{~cm})$ para circunferência abdominal (Tabela 2).
Quanto ao controle da pressão arterial, segundo observado, $20(44 \%)$ participantes possuíam níveis pressóricos nas faixas de normal e ótima, enquanto 11 (22\%) apresentavam-se com níveis na faixa limítrofe e 18 (37\%) participantes, mesmo com a terapêutica, permaneciam com níveis de pressão elevados (Tabela 3).

Tabela 2: Distribuição dos pacientes com hipertensão arterial segundo dados antropométricos. Centro de Referência em Assistência Social de Fortaleza-CE, 2009

\begin{tabular}{lllllll}
\hline Dados Antropométricos & N & Média & DP1 & P 25 & P 50 & P75 \\
\hline IMC2 & 49 & 28 & 3,9 & 24,35 & 28,7 & 30,55 \\
Circunferência Abdominal & 49 & 90 & 10 & 92,00 & 98,00 & 106,50 \\
RCQ $^{3}$ & 49 & 0,9 & 0,1 & 0,89 & 0,93 & 0,98 \\
Prega Tricipital & 49 & 18,9 & 6,1 & 14,15 & 18,30 & 21,95 \\
Prega Subescapular & 49 & 22,3 & 7,4 & 16,10 & 23,60 & 26,95 \\
\hline
\end{tabular}

${ }^{1}$ DP: Desvio-Padrão; ${ }^{2}$ IMC: Índice de Massa Corporal; ${ }^{3}$ RCQ: Relação Cintura-Quadril.

Tabela 3: Caracterização dos níveis de pressão arterial dos participantes avaliados. Centro de Referência em Assistência Social de Fortaleza-CE, 2009

\begin{tabular}{|c|c|c|c|c|c|c|}
\hline Classificação dos níveis pressóricos & & & $\mathrm{N}$ & & $\%$ & \\
\hline Ótima & & & 4 & & 8 & \\
\hline Normal & & & 16 & & 33 & \\
\hline Limitrofe & & & 11 & & 22 & \\
\hline Hipertensão estáqio 1 & & & 1 & & 2 & \\
\hline Hipertensão estáaio 2 & & & 3 & & 6 & \\
\hline Hipertensão estáaio 3 & & & 2 & & 4 & \\
\hline Hipertensão isolada & & & 12 & & 25 & \\
\hline \multirow[t]{2}{*}{ Total } & & & 49 & & 100 & \\
\hline & N & Média & $\mathrm{DP}^{1}$ & P 25 & P 50 & P 75 \\
\hline Grau de Adesão Terapêutica & 41 & 7,7132 & 1,24088 & 6,6200 & 8,1200 & 8,8250 \\
\hline \multicolumn{7}{|l|}{ Correlação de Pearson } \\
\hline Média da PAS 2 de 3 encontros & $-0,675$ & & & & & \\
\hline Média da $\mathrm{PAD}^{3}$ de 3 encontros & $-0,593$ & & & & & \\
\hline
\end{tabular}

${ }^{1}$ DP: Desvio-Padrão; 2 PAS: Pressão Arterial Sistólica; ${ }^{3}$ PAD: Pressão Arterial Diastólica.

Em relação aos hábitos de vida adotados por esses indivíduos, percebeu-se o seguinte: $28(57 \%)$ referiram uso de dieta hipossódica; 26 (53\%) consumiam verduras com frequência; 32 (65\%) ingeriam frutas no mínimo uma vez por dia (Tabela 4).

Sobre os hábitos de tabagismo e etilismo, 46 (94\%) não eram tabagistas e $44(90 \%)$ não consumiam bebidas alcoólicas. Referente ao uso de outras medicações, 25 (51\%) participantes utilizavam fitoterápicos, dos quais o chá de ervas foi a mais referida e apenas uma participante adotava reposição hormonal com estrógenos. Ademais, 25 (51\%) entrevistados afirmaram controlar 0 estresse em situações desencadeantes e 24 (49\%) disseram executar alguma atividade física, predominantemente caminhada, com duração média de sessenta minutos, realizada quatro vezes por semana. No grupo em estudo, a adesão a essa terapêutica não medicamentosa é explicada pela grande participação deles no Projeto Saúde do Corpo de Bombeiros realizado em praças públicas da cidade por oficiais da entidade com vistas à promoção de exercícios físicos na população (Tabela 4). 
Tabela 4: Distribuição dos pacientes com hipertensão arterial segundo os hábitos de vida. Centro de Referência em Assistência Social de Fortaleza-CE, 2009

\begin{tabular}{|c|c|c|}
\hline Variáveis & $\mathrm{N}$ & $\%$ \\
\hline \multicolumn{3}{|c|}{ Dieta hipossódica } \\
\hline Sim & 28 & $57 \%$ \\
\hline Não & 21 & $43 \%$ \\
\hline Total & 49 & $100,0 \%$ \\
\hline \multicolumn{3}{|c|}{ Consumo de verduras } \\
\hline Sim & 26 & $65 \%$ \\
\hline Não & 23 & $35 \%$ \\
\hline Total & 49 & $100,0 \%$ \\
\hline \multicolumn{3}{|c|}{ Consumo de frutas } \\
\hline Sim & 32 & $65 \%$ \\
\hline Não & 17 & $35 \%$ \\
\hline Total & 49 & $100,0 \%$ \\
\hline \multicolumn{3}{|c|}{ Tabagismo } \\
\hline Sim & 3 & $6 \%$ \\
\hline Não & 46 & $94 \%$ \\
\hline Total & 49 & $100,0 \%$ \\
\hline \multicolumn{3}{|c|}{ Ingesta de álcool } \\
\hline Sim & 5 & $10 \%$ \\
\hline Não & 44 & $90 \%$ \\
\hline Total & 49 & $100,0 \%$ \\
\hline \multicolumn{3}{|c|}{ Controle do estresse } \\
\hline Sim & 25 & $51 \%$ \\
\hline Não & 24 & $49 \%$ \\
\hline Total & 49 & $100,0 \%$ \\
\hline \multicolumn{3}{|c|}{ Atividade Física } \\
\hline Sim & 24 & $49 \%$ \\
\hline Não & 25 & $51 \%$ \\
\hline Total & 49 & $100,0 \%$ \\
\hline
\end{tabular}

No relacionado ao uso de medicamentos antihipertensivos, 23 (47\%) utilizavam apenas uma classe de medicamento, adotando, desta forma, a monoterapia. As classes mais utilizadas para este tratamento foram: betabloqueador, 4\%; antagonista de receptor da angiotensina II, 4\%; inibidor da Enzima Conversora de Angiotensina II (ECA), 44\%; e diurético, $48 \%$ (Tabela 5).

Quanto à terapia combinada, o uso de mais de uma classe de medicamentos era seguida por $26(53 \%)$ pessoas. A associação entre o uso de dois fármacos era a mais frequente, 18 (69\%), correspondendo em 11 (61\%) das situações a associação de diuréticos e inibidores da ECA. A combinação de quatro ou cinco classes de fármacos foi observada em apenas 2 $(8 \%)$ integrantes da população (Tabela 5).

Tratando-se isoladamente cada classe de medicamentos, sobressaíram: diuréticos, 35 (72\%); inibidores da ECA, 27 (55\%); betabloqueador, 11 (22\%); vasodilatador, 6 (12\%); bloqueador de canal de cálcio, $3(6 \%)$; e antagonista de receptor de angiotensina II, 1 (2\%) (Tabela 5).
Efeitos adversos foram relatados por 24 (49\%) dos indivíduos. Como observado, mencionaram-se 35 diferentes reações adversas, sendo as mais frequentes tosse seca, dormência, boca seca e câimbras musculares, poliúria e tontura (Tabela 5).

No concernente à associação das reações adversas aos grupos de medicamentos, perceberam-se a relação delas com pelo menos uma das classes entre os inibidores da ECA, diuréticos, betabloqueadores, vasodilatadores, bloqueadores de canal de cálcio e antagonistas de receptor da angiotensina II. Todas essas classes foram citadas no estudo por pelo menos um indivíduo.

0 grau de adesão avaliado segundo 0 instrumento proposto por Moreira $(2003)^{6}$ foi obtido em um segundo encontro ao qual não compareceram oito dos participantes do estudo. Os 41 clientes avaliados apresentaram uma média de adesão de 7,71 ( \pm 1,24). 0 grau de adesão só denotou associação significativa com as médias de pressão arterial sistólica e pressão arterial diastólica obtidas em três encontros, de acordo com o Teste de Pearson (Tabela 3). 
Segmento terapêutico em portadores de hipertensão arterial

Vito AF, Monteiro FPM,Morais HCC, Vasconcelos JDP, Lopes MVO,Araújo TL

Tabela 5: Distribuição dos pacientes com hipertensão arterial segundo a terapêutica farmacológica e seus efeitos. Centro de Referência em Assistência Social de Fortaleza-CE, 2009

\begin{tabular}{lcc}
\hline Variáveis & N & $\%$ \\
\hline Esquemas Terapêuticos Farmacológicos & & \\
Monoterapia & 23 & $47 \%$ \\
Terapia Combinada & 26 & $53 \%$ \\
\hline Total & 49 & $100 \%$ \\
\hline Classes de Medicamentos utilizados & & \\
Diurético & 35 & $72 \%$ \\
Inibidor da ECA & 27 & $55 \%$ \\
Betabloqueador & 11 & $22 \%$ \\
Vasodilatador & 6 & $12 \%$ \\
Bloqueador de canal de cálcio & 3 & $6 \%$ \\
Antagonista de receptor da & 11 & $2 \%$ \\
angiotensina II & & \\
Efeitos Adversos & & \\
Sim & 24 & $49 \%$ \\
Não & 25 & $51 \%$ \\
\hline Total & 49 & $100 \%$ \\
\hline Efeitos Adversos relatados & & \\
Poliúria & 7 & $29 \%$ \\
Tontura & 7 & $29 \%$ \\
Tosse seca & 6 & $25 \%$ \\
Dormência & 6 & $20 \%$ \\
Boca seca & 5 & \\
Câimbras musculares & 5 & \\
\hline
\end{tabular}

\section{DISCUSSÃO}

Os 49 participantes do estudo constituíram-se predominantemente de idosos. No referente ao quantitativo de participantes deste estudo, considera-se que o número reduzido e a especificidade do local investigado limitaram a representatividade dos achados. Em face disso, sugere-se que outros estudos com amostras maiores sejam executados em diferentes localidades.

Nesta ocasião, a faixa etária representa um importante fator de risco para as doenças cardiovasculares. ${ }^{1}$ Isso ocorre em virtude das alterações próprias do envelhecimento, evidenciadas pela modificação da complacência arterial e das alterações na parede dos vasos. Portanto, são frequentes complicações como doença arterial coronária, doença cerebrovascular, insuficiência cardíaca, doença vascular periférica, disfunção diastólica, entre outras. $^{8}$

Nesta faixa etária, existe um predomínio de diagnosticados do sexo feminino. De modo geral, até os 40 anos, os níveis pressóricos são mais elevados nos homens, mas a partir desta faixa etária as mulheres passam a ser mais acometidas pela hipertensão por causa da instalação do período do climatério com a consequente perda da proteção dos hormônios femininos sobre o sistema cardiovascular. ${ }^{9}$ Esses têm ação favorável sobre o perfil lipídico, além da ação antiagregante plaquetária e vasodilatadora. ${ }^{10} 0$ maior número de mulheres diagnosticadas também pode ser influenciado pela preocupação dessas com a saúde, o que explicaria a razão de procurarem mais cedo os serviços de saúde. ${ }^{11}$

Segundo a literatura, a raça negra ou parda sugere um aumento na probabilidade de ocorrência de lesões em órgão-alvo, referente à hipertensão arterial. ${ }^{12}$ No entanto, pela dificuldade de classificação de cor de pele e pela intensa miscigenação observada no Brasil, é mais frequente os indivíduos se classificarem como pardos, conforme verificado.

Outro agravante é a falta de convivência com companheiros em união estável em face da possibilidade de comprometer a adesão ao tratamento, uma vez que a relação com outra pessoa funciona como facilitadora do 
processo de adesão ao tratamento, por representar, comumente, apoio no processo de autocuidado e melhora o convívio social. ${ }^{13}$

A adesão ao tratamento também pode ser prejudicada pelo baixo nível de escolaridade, pois a leitura e a compreensão de receitas e orientações são fundamentais para aumentar a adesão. Um baixo nível de escolaridade pode contribuir para o agravamento da hipertensão, porquanto, associado a fatores socioeconômicos e culturais, pode dificultar a adesão ao tratamento, bem como a manutenção de comportamentos e hábitos de vida saudáveis. $^{13}$

De modo geral, os níveis socioeconômicos mais baixos estão associados a maiores índices de pressão arterial elevados, em virtude de fatores como dificuldade de acesso aos serviços de saúde, hábitos dietéticos e estresse psicossocial. ${ }^{1}$ Ademais, em alguns casos, o fármaco de primeira escolha para o tratamento não é fornecido pelo sistema público de saúde, comprometendo a terapêutica de melhor eficácia naqueles casos em que o paciente não possui condições financeiras para comprá-lo.

Conforme se afirma, o índice de massa corporal é utilizado para avaliar se a pessoa apresenta excesso de peso, enquanto a medida da cintura e sua relação com o quadril caracterizam a proporção de depósito de gordura nas duas regiões. Essas medidas são úteis para 0 monitoramento dos pacientes, e, quanto maiores os índices, maiores os riscos cardiovasculares. ${ }^{14}$ As pregas subcutâneas refletem a espessura da pele e tecido adiposo subcutâneo, os quais estimam as reservas de gordura do corpo.

Complementarmente ao IMC na avaliação das alterações metabólicas que desencadeiam a obesidade e suas complicações, existe a RCQ. Logo, quando elevada, está correlacionada com um perfil lipídico adverso em hipertensos. Recomenda-se que a RCQ deve estar abaixo de 0,90 em homens sem sobrepeso e abaixo de 0,85 em mulheres com sobrepeso. ${ }^{14}$

Os participantes do estudo em discussão apresentaram, portanto, alto risco cardiovascular uma vez que, apesar do maior número de participantes do sexo feminino, a média encontrada situou-se acima do valor considerado limítrofe para o sexo masculino. Corrobora este resultado uma pesquisa realizada com portadores de angina instável, que apresentaram valores acima do esperado para ambos os sexos para a relação cintura quadril. ${ }^{15}$

Torna-se evidente, portanto, a necessidade da adoção nos serviços de saúde da avaliação do IMC, da RCQ, das pregas cutâneas e da circunferência abdominal, com vistas a obter maior suporte para identificação de alterações, assim como oferecer mais dados para controle do seguimento terapêutico por parte do próprio cliente.

A pressão sistólica é o mais importante parâmetro para predizer o risco de eventos cardiovasculares dos pacientes portadores de hipertensão arterial, particularmente após os 60 anos de idade. ${ }^{16}$ Quanto à classificação desse grupo, a maioria apresentou valores da pressão arterial considerados controlados, com $63 \%$ classificados em valores ótimos, normais e limítrofes. Sendo o valor da PA o principal aspecto a ser considerado quando se trata de avaliação do resultado ou eficácia do tratamento para hipertensão, pode-se afirmar que a terapêutica implementada e o seguimento desta estavam satisfatórios nessa clientela.

Em contrapartida, ao comparar os resultados atuais com os obtidos em estudo anterior ${ }^{13}$ no mesmo local, verificou-se queda de $25 \%$ no grupo de pessoas com PA classificada como ótima ou normal. Ainda relacionando com o referido estudo, pôde-se perceber também o aumento da população com PA limítrofe, hipertensão estágio II, estágio III e hipertensão arterial sistólica isolada. Cabe destacar que os participantes não obrigatoriamente foram os mesmos, sobretudo porque os estudos foram realizados em períodos diferentes de tempo.

Em pesquisa ${ }^{17}$ referente à avaliação da pressão arterial de 462 pessoas com diagnóstico de hipertensão e que faziam tratamento farmacológico, somente $22,3 \%$ dos pacientes estavam com pressão arterial controlada, índice bem inferior ao encontrado no presente estudo. Esse fato pode ser decorrente da população mais específica do estudo em elaboração, cujos participantes recebiam constantemente orientações relacionadas à saúde.

Neste caso, a terapêutica não farmacológica empregada baseava-se no uso restrito de sal, consumo de frutas e verduras diariamente na alimentação, desestímulo ao uso de álcool e abolição do fumo, controle do estresse e a prática de exercícios físicos. A maior parte da população estudada pertenceu ao grupo com estilo de vida saudável com relevante adesão à terapêutica, pois os fatores previamente mencionados que compreendem o tratamento não farmacológico para hipertensão podem não só reduzir o uso de anti-hiper tensivos como também melhorar o perfil dos outros fatores de risco cardiovascular e a qualidade de vida dos pacientes. ${ }^{8}$

Segundo evidenciado, parcela significativa dos idosos afirmou seguir dieta hipossódica, com redução da ingestão de cloreto de sódio na alimentação. De acordo com isso, conforme a literatura aponta, a pressão arterial varia diretamente com a quantidade de sal consumida. Portanto, os efeitos do consumo excessivo de sal podem ser observados em toda a população, porém, mais expressivamente, nos portadores de hiper tensão arterial. Dessa forma, a redução do consumo diário de sal deve ser incentivada, pois mesmo pequena e desacompanhada de perda de peso promove discreta redução dos níveis de pressão arterial. Os participantes do estudo demonstraram conhecimento sobre os efeitos negativos do sal na pressão arterial. 
Como adverte a literatura, a dieta recomendada para portadores de hiper tensão arterial deve incentivar o consumo de frutas e verduras e reduzir a ingestão de alimentos gordurosos que poderão aumentar os índices séricos de colesterol, representando fator de risco para o desenvolvimento da aterosclerose. ${ }^{1}$ Apesar de a maioria dos pacientes referir consumir frutas e verduras, acreditase que a quantidade ingerida não é equivalente à recomendada, simbolizando a grande dificuldade na mudança dos hábitos alimentares para os participantes em discussão.

Conforme os dados, o princípio do tratamento não farmacológico que apresentou maior adesão foi a abstinência ao etilismo e tabagismo. Este fato constitui um grande avanço no controle da PA, uma vez que estes fatores aumentam os riscos de doenças cardiovasculares. 0 etanol exerce efeito deletério sobre o sistema cardiovascular, provocando elevação dos níveis de pressão, principalmente da PAS. Já a nicotina estimula a liberação da norepinefrina das terminações nervosas adrenérgicas e, em associação com outros componentes do cigarro, lesiona o endotélio dos vasos sanguíneos, provocando arteriosclerose. Esta aumenta a resistência vascular periférica e contribui para o aumento dos níveis de pressão arterial. ${ }^{5}$

Ainda como mostra a literatura, as emoç̃es podem elevar a pressão de forma aguda, mas quando as situações conflitantes são prolongadas e recorrentes podem manter os valores elevados de forma persistente. ${ }^{5} 0$ controle do estresse naquelas situações que incitam tal comportamento representa uma atitude importante para a manutenção de índices de pressão arterial dentro dos valores de normalidade. Tal comportamento é procurado pela maioria dos participantes do estudo.

Para todos os portadores de hipertensão arterial, mesmo aqueles em uso de tratamento medicamentoso, recomenda-se o exercício físico. 0 treinamento físico moderado e regular promove queda da pressão ar terial por reduzir a atividade simpática e do tônus simpático cardíaco, além de ajudar na adequação do peso corporal. ${ }^{18}$

Contudo, o tratamento farmacológico para hipertensão arterial deve ser instituído naqueles indivíduos que, mesmo com as devidas modificações nos hábitos de vida, persistem com valores de pressão arterial acima do recomendado. Deve ser iniciado sempre que a pressão sistólica for $\geq 160 \mathrm{mmHg}$ e/ou a pressão diastólica for $\geq$ $100 \mathrm{mmHg}$. Além dessas situações, também deve ser considerado para os casos em que após seis meses de tratamento não farmacológico o paciente continue apresentando valores de PAS entre 140 e $150 \mathrm{mmHg}$ e/ou PAD acima de $90 \mathrm{mmHg}{ }^{1}$

Para monoterapia inicial, especialmente em pacientes com hipertensão arterial estágio I, qualquer medicamento anti-hipertensivo pode ser utilizado, com exceção dos vasodilatadores de ação direta, pois esses podem provocar retenção hídrica e taquicardia reflexa quando utilizados isoladamente. Em casos de estágio II ou III, o uso de associações de medicamentos deve ser considerado mesmo como terapia inicial. A terapia combinada está indicada quando outro esquema não foi suficiente para atingir as reduções de pressão previstas ou diante da necessidade de controle de pressão mais rigoroso. ${ }^{1}$

Entre as terapias combinadas com uso de dois fármacos, destacou-se a associação de diurético e inibidor da ECA, identificada em $61 \%$ da população. Esta associação apresenta boa eficácia e tolerabilidade, além de baixo custo. Isso pode justificar a preferência pela terapia com associação das referidas drogas. ${ }^{1}$

Segundo observou-se, $26 \%$ das pessoas em uso de esquema farmacológico monoterápico não tinham pressão arterial controlada. Já em relação às que usavam terapia combinada, $50 \%$ estavam com pressão arterial alterada. Destaque-se o seguinte: as associações medicamentosas têm sido evitadas, visto que, mesmo em pacientes com PA elevada, há indicação do uso de apenas um fármaco. Outro dado relevante foi o fato de que a maior prevalência de pessoas com PA elevada está entre os que já realizam terapia combinada, pois neste grupo o controle pressórico deveria ser mais rigoroso por se tratar de pessoas com terapêutica mais complexa.

Conforme os resultados de estudo realizado em $2007^{17}$, o esquema mais utilizado pelas pessoas era a monoterapia (66\%), e a droga mais prescrita foi representada pela classe dos inibidores da ECA (65\%). Pode-se ainda identificar no mesmo estudo que o melhor controle de pressão arterial, entre aqueles com esquema monoterápico, foi dos usuários de diurético.

Os diuréticos tiazídicos são os fármacos de primeira escolha no tratamento da $\mathrm{HA}$ e trazem importantes benefícios cardiovasculares, podendo contribuir no tratamento da osteoporose. Com eficácia semelhante, têm-se os inibidores da ECA, capazes de reduzir a mortalidade cardiovascular, com vantagens para diabéticos e portadores de nefropatias. Demais fármacos possuem maiores especificidades para indicação de uso, como no caso de antagonista de receptor de angiotensina II com menores efeitos adversos. Os bloqueadores de canal de cálcio estão mais indicados para pessoas com maior risco de acidentes vasculares cerebrais e os medicamentos betabloqueadores, para pacientes com insuficiência cardíaca, taquiarritmias e tremores. ${ }^{17}$

Tendo em vista que a hipertensão cursa, na maioria dos casos, de forma assintomática, fica evidente, nessa situação, a importância negativa dos efeitos 
adversos, principalmente em relação à adesão à terapêutica proposta. ${ }^{1}$ Segundo estudo realizado em $2007^{17}, 9,9 \%$ daqueles que não seguiam o tratamento farmacológico conforme as prescrições atribuíram o fato aos efeitos colaterais da terapêutica medicamentosa.

0 grau de adesão verificado mediante utilização da escala proposta por Moreira (2003) mostra-se satisfatório, com média de 7,71, pertencente à categoria de não adesão leve. Destaca-se, porém, o fato de nenhum paciente atingir a categoria de adesão ideal. Em contrapartida, também não se encontrou não adesão grave. Entretanto, se comparado com estudo semelhante efetuado em $2007^{13}$ e desenvolvido no mesmo local, há uma redução da adesão terapêutica, pois, no referido estudo, a média do grau de adesão encontrada foi de 8,21 ( $\pm 0,956)$, com uma paciente alcançando a pontuação máxima de adesão ideal.

\section{CONCLUSÕES}

Como os resultados demonstraram, os participantes seguem parte do tratamento anti-hipertensivo, adaptandoo a sua realidade. Isso provoca uma adesão incapaz de reduzir os níveis de pressão arterial de forma satisfatória. Contudo, os entrevistados buscaram iniciativas positivas para conhecer e melhorar seu estado de saúde. Os níveis de pressão arterial e graus de adesão satisfatórios corroboram tal afirmação. A partir disso, o enfermeiro deve conhecer as características predominantes do grupo de portadores de hipertensão arterial para facilitar 0 planejamento das intervenções propostas ao tratamento, além dos devidos ajustes direcionados aos aspectos da clientela geradores de uma menor taxa de adesão. Com o auxílio da educação em saúde contínua e reforço positivo constante, é possível melhorar os resultados encontrados e aumentar a adesão terapêutica.

\section{REFERÊNCIAS}

1. Diretrizes Brasileiras de Hipertensão Arterial, 5. Rev Bras Hipertens. 2006; 13(4): 260-312.

2. Araújo GBS, Garcia TR. Adesão ao tratamento anti-hipertensivo: uma análise conceitual. Rev Eletr Enferm. 2006; 8(2): 259-72.
3. Lyra Júnior DP, Amaral RT, Veiga EV, Cárnio EC, Nogueira MS, Pelá IR. A farmacoterapia no idoso: revisão sobre a abordagem multiprofissional no controle da hipertensão arterial sistêmica. Rev Latino-am Enfermagem. 2006; 14(3): 435-41.

4. Saraiva KRO, Santos ZMSA, Landim FLP, Teixeira AC. Saber do familiar na adesão da pessoa hipertensa ao tratamento: análise com base na educação popular em saúde. Texto\& Contexto Enferm. 2007; 16(2): 263-70.

5. Mantovani MF, Mottin JV, ULbrich EM, Pinotti S. Caracterização dos usuários e o conhecimento sobre a hipertensão arterial. Online Braz J Nurs. 2008; 7(2): 61-69.

6. Moreira TMM. Tecnologia de cuidado na busca da adesão ao tratamento da hipertensão arterial: desenvolvimento e avaliação de uma experiência em Fortaleza-Ceará [tese]. Fortaleza: Departamento de Enfermagem, Faculdade de Farmácia, Odontologia e Enfermagem/ UFC; 2003.

7. Ministério da Saúde (BR). Conselho Nacional de Saúde. Resolução $n^{\circ} 196$, de 10 de outubro de 1996. Diretrizes e normas regulamentadoras de pesquisas envolvendo seres humanos. Bioética 1996; 4(2): 15-25.

8. Miranda RD, Perrotti TC, Bellinazzi VR, Nóbrega TM, Cnedoroglo MS, Toniolo Neto J. Hipertensão arterial no idoso: peculiaridades na fisiopatología, no diagnóstico e no tratamento. Rev Bras Hipertens. 2003; 9(3); 293-300.

9. Monteiro PC, Santos FS, Fornazari PA, Cesarino CB. Características biossociais, hábitos de vida e controle da pressão arterial dos pacientes em um programa de hipertensão. Arq Cienc Saude. 2005; 12(2): 73-9.

10. Freitas F, Menke CH, Rivoire WA, Passos EP. Rotinas em ginecologia. $5^{\mathrm{a}}$ ed. Porto Alegre: ArtMed; 2006. 584p.

11. Santos ZMSA, Frota MA, Cruz DM, Holanda SDO. Adesão do cliente hipertenso ao tratamento: análise com abordagem interdisciplinar. Texto\&Contexto Enferm. 2005; 14(3): 332-40.

12. Katzung BG. Farmacologia básica e clínica. $9^{\mathrm{a} e d .}$ Rio de Janeiro: Guanabara Koogan; 2006. 1008p.

13. Oliveira CJ. Idosos em tratamento farmacológico antihipertensivo: parâmetros para o cuidado clínico de Enfermagem [dissertação]. Fortaleza: Universidade Estadual do Ceará; 2007.

14. Pereira RA, Sichieri R, Marins VMR. Razão cintura/quadril como preditor de hipertensão arterial. Cad Saude Publica. 1999; 15(2): 333-44.

15. Rocha DH, Sousa VEC, Pascoal LM, Montoril MH, Monteiro FPM, Lopes MVO. Troca de gases prejudicada em pacientes com angina instável. Esc Anna Nery. 2009; 13(3): 471-76.

16. Kannel WB, Wolf PA, Daniel LM, Thomas RD, Mcnamara P, Castelli WP et al. Systolic blood pressure, arterial rigidity, and risk of stroke: the Framingham study. JAMA. 1981; 245(12): 1225-229. 
Segmento terapêutico em portadores de hipertensão arterial

Vito AF, Monteiro FPM,Morais HCC, Vasconcelos JDP, Lopes MVO,Araújo TL

17. Mochela EG, Andrade CF, Almeida DS, Tobias AF, Cabral R, Cossetti RD. Avaliação do tratamento e controle da hipertensão arterial sistêmica em pacientes da rede pública em São Luis (MA). Rev Baiana Saude Publica. 2007; 31(1): 90-101.

18. Ferreira Filho C, Meneghini A, Riera ARP, Serpa Neto A, Teixeira GK, Ferreira C. Benefícios do exercício físico na hipertensão arterial sistêmica. Arq Med ABC. 2007; 32: 82-7.

\section{NOTA}

aTrabalho desenvolvido com base no projeto PIBIC-CNPq 2008-2009, processo n ${ }^{0} 117498 / 2008-4$, em Enfermagem da Universidade Federal do Ceará (UFC). 